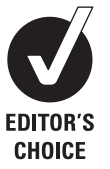

CHOICE

\title{
Co-existent medial collateral ligament injury seen following transient patellar dislocation: observations at magnetic resonance imaging
}

\author{
J F Quinlan, ${ }^{1}$ C Farrelly, ${ }^{2}$ G Kelly, ${ }^{3}$ S Eustace ${ }^{2}$
}

${ }^{1}$ Department of Orthopaedic Surgery, Cappagh National Orthopaedic Hospital, Finglas, Dublin 11, Republic of Ireland 2Department of Radiology, Cappagh National Orthopaedic Hospital, Finglas, Dublin 11, Republic of Ireland;

${ }^{3}$ School of Mathematical Sciences, University College Dublin, Belfield, Dublin 4, Republic of Ireland

\section{Correspondence to}

Dr J Quinlan, 35 Rockford

Manor, Stradbrook, Blackrock, Co Dublin, Republic of Ireland; johnfquinlan@yahoo.com

Accepted 28 October 2008 Published Online First 16 November 2008

\section{ABSTRACT}

This study reports on a series of patients who were diagnosed as having had a transient lateral patellar dislocation by magnetic resonance imaging (MRI). The images were reviewed with specific reference to the medial collateral ligament (MCL), a heretofore undescribed concomitant injury. Eighty patients were diagnosed on MRI as having had transient lateral patellar dislocation. Their mean age was 23.9 years (SD 7.5). Forty patients $(50.0 \%)$ had co-existent MCL injuries. These injuries were classified as grade $1(\mathrm{n}=20)$, grade $2(n=17)$ and grade $3(n=3)$. These results suggest that MCL injury commonly accompanies transient lateral patella dislocation, most likely due to a shared valgus injury. It appears to occur more commonly in male patients and if unidentified may explain both delayed recovery and persistent morbidity in more severe cases. In this setting, without specifically excluding co-existent MCL injury, the current vogue for early rehabilitation should be adopted with caution.

Patellar dislocation accounts for $2-3 \%$ of all knee injuries $^{1}$ and is seen as the second most common cause of knee haemarthrosis. ${ }^{2}$ Despite this, its pathophysiology is poorly understood and its management continues to remain controversial. The main ongoing problem is recurrence but it is still far from certain which if any population is most at risk for this and if surgery diminishes this risk. ${ }^{3}$

Traditionally, this is an injury that has been associated with women although this has been challenged in some reports. ${ }^{45}$ This association is felt to be due to a relatively increased genu valgum seen in women ${ }^{67}$ although it is unclear if any particular sport represents a specific risk. ${ }^{8}$ Furthermore, it has been described mainly with patients in their second decade of life, ${ }^{59}$ although Atkin et $a l^{5}$ do stress the potential bias in such demographic statements. It is possible that decreasing neuromuscular control over the lower limb ${ }^{10} 11$ may contribute to a patient's susceptibility but it would appear difficult to provide agespecific aetiologies. However, it is also an injury that can be transient in nature, with patients unaware it has happened. Indeed, magnetic resonance imaging (MRI) can often offer the first diagnosis with its characteristic pattern of lateral femoral condyle bone bruising. ${ }^{12}$

The established risk factors for patellar dislocation are many and well documented. These include trochlear dysplasia, ${ }^{13}$ medial patellofemoral ligament insufficiency, ${ }^{14}$ patella alta, ${ }^{15}$ abnormal $\mathrm{Q}$ angle, ${ }^{16}$ genu valgum, ${ }^{17}$ generalised ligamentous laxity $^{18}$ and iliotibial band dysfunction. ${ }^{19}$ A relationship between patellar dislocation and patellar maltracking is also quoted, and this is felt to be due to an imbalance between the vastus medialis and vastus lateralis. ${ }^{20}{ }^{21}$ Unequal forces across the knee have also led to eccentric histological changes seen in patients with chronic patellar tendinitis ${ }^{22}$ and this may also be an association with patellar dislocation.

Treatment of this condition can be simplified into conservative and surgical modalities. Redislocation rates with these methods have been quoted at $0-47 \%$ and $0-32 \%$, respectively. ${ }^{1}$ There are currently over 100 documented surgical procedures described, and apart from recurrence complications have only been associated with operative interventions. ${ }^{23}$ In relation to conservative management, the emphasis is placed on aggressive, early mobilisation of the patient. ${ }^{24}$

Stefancin and Parker ${ }^{1}$ produced a meta-analysis of the 70 published papers on this subject in 2007. The authors noted that due to the multifactorial element of the condition and debate about management, multiple confounders existed in many studies. However, in an attempt to summarise treatment, they advocated conservative management, except in cases when associated with osteochondral fractures, substantial disruption of medial patellar stabilisers, lateral subluxation of the patella compared with the contralateral knee, second dislocation or failure to respond to rehabilitation. ${ }^{1}$

The medial patellar stabilisers are both static, such as the inherent bony anatomy ${ }^{25}$ and dynamic, namely the medial retinaculum, ${ }^{26}$ medial patellofemoral ligament (which accounts for $60 \%$ of medial stability $)^{27}$ and the vastus medialis obliquus (VMO) ${ }^{28}$ However, to date, the authors are unaware of any studies looking at the medial collateral ligament (MCL) and its association with this injury complex in patellar dislocation.

The aim of this MRI-based study was to establish an incidence of MCL injury in patients with transient patellar dislocation and to examine if there was a significant age or gender association.

\section{MATERIALS AND METHODS}

During the study period from November 2001 to April 2008 inclusive, 80 patients were diagnosed with transient patellar dislocation, confirmed by MRI scanning. In all cases, the radiological diagnosis correlated with the clinical findings in these patients. No patient had any previous diagnoses of patellar dislocation. 
All patients were imaged in a Philips new generation Gyroscan Intera Tesla (T) scanner (Koninklijke Philips Electronics Ltd, Eindhoven, The Netherlands) and had coronal short T1 inversion recovery (STIR), coronal T1 and sagittal and axial T2 images performed.

The resultant scans were reported on by a musculoskeletal fellowship-trained radiologist. The radiological diagnosis of transient patellar dislocation was confirmed by the presence of the characteristic countercoup pattern of lateral femoral condyle bone bruising (fig 1). Following this, the status of the other structures in the knee was commented upon.

In particular, the status of the MCL was noted. Injuries to this structure were classified as grades $1-3$ as per the classification of Schweitzer et al. ${ }^{29}$ This system defines grade 1 injuries as having fluid on the inner or outer aspect of the superficial fibres. Grade 2 injuries show fluid on both sides of the ligament with or without internal foci of high signal but without discontinuity, whereas grade 3 injuries are classified as having complete disruption/discontinuity of the ligament (table 1).

Statistical analysis was performed initially between the noinjury and the injury group using a two-sample t-test. Then, looking specifically at the injury group, a linear model was fitted to age with covariates gender, injury group and the interaction gender by injury group. The first term assesses the effect of gender on age, the second the effect of injury group on age and the last term allows for a different effect of injury group between men and women. In an alternative analysis, as the number with grade $3 \mathrm{MCL}$ injuries was so small, the Wilcoxon rank sum test was used in comparisons involving this group; $p$ values of $p<0.05$ were regarded as statistically significant. All analyses were carried out using SAS version 9.1.3.

\section{RESULTS}

Eighty patients imaged during the study period had a radiological diagnosis of transient patellar dislocation. This group consisted of 66 men and 14 women. The mean age of the total study cohort was 23.9 years (SD 7.5, range 11-60). The mean ages of the male group was 23.7 years (SD 5.9, range 14-41) and of the female group was 25.0 years (SD 12.9, range 11-60).

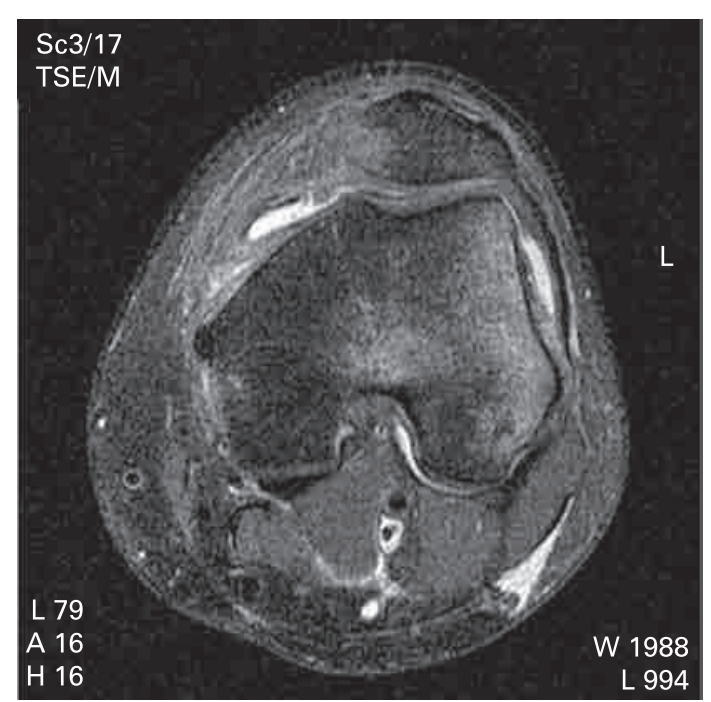

Figure 1 Axial T2 image of the knee showing lateral femoral condyle and medial patellar facet bone bruising consistent with a diagnosis of transient patellar dislocation.
Table $1 \mathrm{MRI}$ findings seen in injuries of the MCL

\begin{tabular}{ll}
\hline Grade & Findings \\
\hline 1 & Fluid on either the inner or the outer aspect of the superficial fibres \\
2 & $\begin{array}{l}\text { Fluid on both sides of the ligament with or without internal foci of high signal } \\
\text { but without discontinuity }\end{array}$ \\
3 & Complete disruption/discontinuity of the ligament
\end{tabular}

Reproduced from Schweitzer et $\mathrm{al}_{1}{ }^{29}$ with permission. MCL, medial collateral ligament; $\mathrm{MRI}$, magnetic resonance imaging.

All 80 patients had bone bruising of their lateral femoral condyle in a pattern consistent with transient patellar dislocation. In addition, patellar derangements were described in 40 of the patients. These took the form of bone bruising to the medial facet $(n=30)$ and chondral injuries $(n=10)$. The chondral injuries were subdivided according to their location and consisted of the junction of the medial and lateral facets $(\mathrm{n}=3)$, the lateral facet $(\mathrm{n}=3)$, the medial facet $(\mathrm{n}=2)$. In the other two cases, medial facet bone oedema was observed in association with a chondral injury to the junction of the medial and lateral facet $(n=1)$ and to the medial facet $(n=1)$.

Of specific relevance to this study was the MCL and any attendant damage to it. This is an association that has not previously been described to the authors' best knowledge. The MCL was found to be injured in 40 cases. The mean age of patients with MCL injuries was 23.5 years (SD 5.1, range 1536). There were 36 men with a mean age of 23.8 years (SD 5.1, range 15-36) and four women with a mean age of 20.8 years (SD 4.6, range 15-25).

Looking at the groups overall, the non-MCL injury group had a mean age of 24.3 years (SD 9.4, range 11-60). This compares with a mean age of 23.5 years (SD 5.1, range 15-36) for the $\mathrm{MCL}$ injury group. When these groups were compared, no significant differences were found among men $(p=0.85)$, women $(p=0.46)$ or overall $(p=0.63)$. Moreover, there was no significant difference between the male no-injury and female no-injury groups $(p=0.33)$.

Out of the 40 patients with MCL damage, 20 were classified as grade 1 (fig 2). The mean age of this group was 21.6 years (SD 5.1 , range 15-36). There were 17 male patients with a mean age of 21.7 years (SD 5.3, range 15-36). The remaining three female patients had a mean age of 19.3 years (SD 4.5, range 15-24).

Grade $2 \mathrm{MCL}$ injuries (fig 3) were described in 17 patients. This group had a mean age of 25.9 years (SD 4.3, range 16-32). Sixteen of these patients were men with a mean age of 26.0 years (SD 4.4, range 16-32). The sole female patient with a grade 2 injury was 25 years of age. There were three male patients in the grade 3 subgroup (fig 4 ). Their mean age was 24.0 years (SD 2, range 22-26).

When the MCL injury group was analysed, a linear model was fitted to age with covariates gender, injury group and gender by injury group. The interaction term gender by injury group was not significant, ie, the age differences between injury groups was the same for men and women $(p=0.81)$. The effect of gender was not significant $(p=0.43)$, ie, there was no significant difference in age between men and women. There was a significant difference between injury groups with patients, with grade $1 \mathrm{MCL}$ injuries having a significantly lower age than those in the grade 2 group $(p=0.005)$, and when adjusted for multiple comparisons (three) $(p=0.015)$. The difference was 4.59 years, with a standard error of 1.54 . In an alternative analysis, as the number with grade $3 \mathrm{MCL}$ damage was so small the Wilcoxon rank sum test was used to compare separately patients in the grade 1 and grade 3 groups and the $p$ 


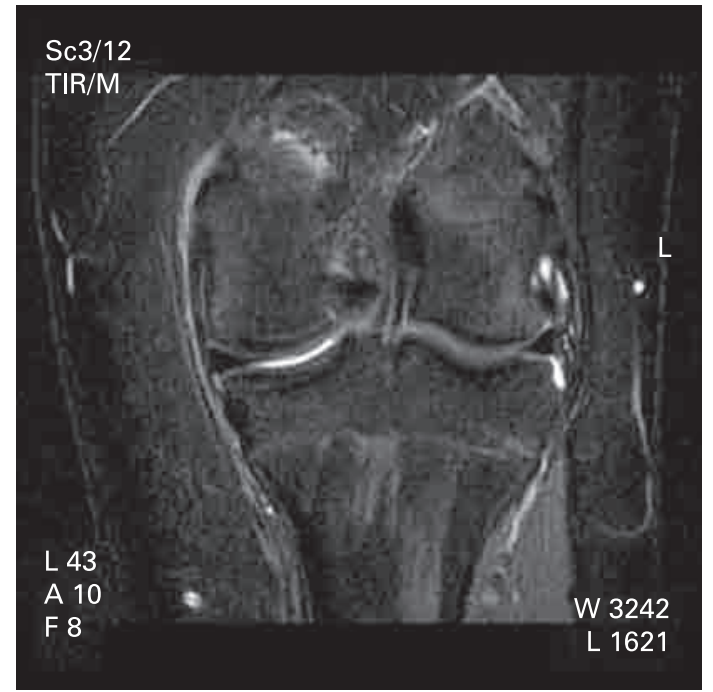

Figure 2 Coronal short T1 inversion recovery image showing a grade 1 medial collateral ligament injury as characterised by fluid on the inner side only of the superficial fibres of the ligament.

value was $p=0.17$. Comparing the grade 2 and grade 3 groups the difference was also not significant $(p=0.33)$.

\section{DISCUSSION}

The mean age of 23.9 years (SD 7.5) in this study as well as the subgroup mean ages of 24.3 years (SD 9.4) in the non-MCL injury group and 23.5 years (SD 5.1) in the patients with MCL injuries is older than the traditionally described second decade. ${ }^{99}$ It is also older than the mean 21.5 years stated in the review by Stefancin and Parker. ${ }^{1}$ Furthermore, the preponderance of 66 men overall and 36 men in the MCL injury group is also in contrast to the majority of the studies on the subject. ${ }^{1}$

Whereas the association between patellar dislocations and MCL injuries has not to the authors' knowledge previously been described, Hunter et al ${ }^{30}$ published a series of surgically repaired MCL injuries and looked at the incidence of vastus medialis

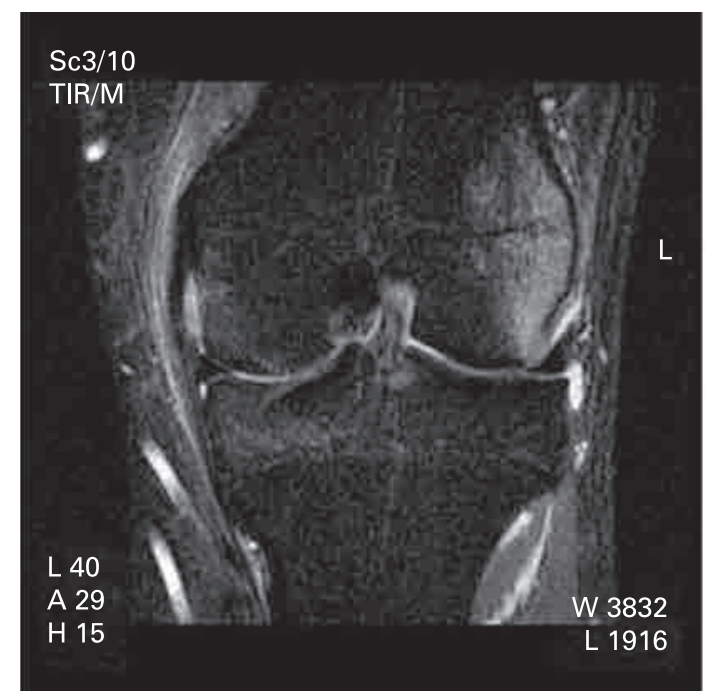

Figure 3 Coronal short T1 inversion recovery image showing a grade 2 medial collateral ligament injury as characterised by fluid on both sides of the superficial fibres of the ligament. The ligament remains in continuity.

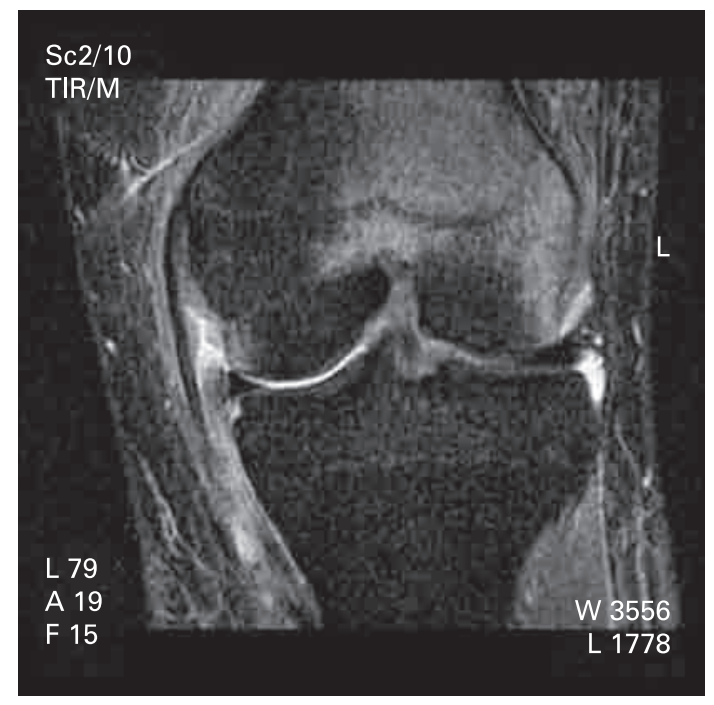

Figure 4 Coronal short T1 inversion recovery image showing a grade 3 medial collateral ligament injury as characterised by fluid on both sides of the ligament associated with a discontinuity of the ligament.

injury. They reported on a consecutive series of 189 moderate and severe MCL injuries that underwent open repair. In their series, they noted a $21 \%$ (40 patients) incidence of VMO disruption. They concluded that a significant relationship exists between acute MCL injury and damage to the VMO muscle. However, no specific correlation is drawn in relation to the reverse of this relationship.

The details of patients' sports were not recorded in this study, although there were no professional sportspeople in the series. However, the three highest participation sports in the reporting country are Gaelic games, soccer and rugby union. Studies of injuries in these sports ${ }^{31-33}$ show that the lower limb accounts for $52.7-87 \%$ of all injuries. In particular, the knee is involved in $12-17 \%$ of injuries. Soft tissue injuries account for $55-81 \%$ of injuries in total. In relation to rugby union injuries, ${ }^{32} 39 \%$ of knee injuries were ligamentous and $75 \%$ of these involved the MCL. It would appear reasonable to presume that this study cohort follows similar patterns.

Due to the retrospective nature of this study, it was not possible to collate data in relation to the contralateral limb or in relation to limb dominance. However, it is worth noting that many players are equally adept at kicking with both feet, with Cromwell et $a^{31}$ citing $34 \%$ of players who kicked with both feet in Gaelic football. This may make limb dominance a moot issue.

It is also worth considering the effect of training and conditioning on the support structures of the knee. Exercise has been seen to increase the mechanical and structural properties of ligaments. ${ }^{34}$ However, overuse can have a detrimental effect on injury status, as seen in other anatomical areas such as the groin ${ }^{35}$ and other sports such as Australian Rules football. ${ }^{36}$ In essence, the cohort in this study is a selfselecting one in that patients with clearly defined patellar pathology have been removed before scanning. The inherent difficulties in specifying exact mechanisms of injury and individual sporting details in groups such as this have been alluded to by others. ${ }^{31} 32$

This study presents a series of injured MCL based on MRI findings. Although all patients underwent clinical examination of their knee before scanning, they were not specifically assessed for this injury. However, many authors have described a high correlation between MRI and clinical findings of MCL damage (7-40 $^{3}$ 


\section{What this study adds}

MCL injury does occur with transient patellar dislocation. It should be examined for, especially in male patients, and treatment of the dislocation may need to be amended if it is found to be present.

while accepting that MRI images may suggest a higher grade of injury than is clinically apparent. ${ }^{41}$

The majority of MCL injuries heal with conservative management and lead to no adverse sequelae for the patient..$^{42}$ However, a small number can go on to cause chronic medial laxity of the knee and require surgical intervention. ${ }^{44}$ Without recognition of the injury, it is difficult to predict which knees will become chronically lax.

In summary, the results of this paper present strong evidence for the existence of a relationship between patellar dislocation and MCL injury. It is to be presumed that while the MCL plays no role in maintaining patellar stability, its role as the primary restraint to valgus in the $k n e e^{45}$ puts it at risk during the mechanism of injury of patellar dislocation. In particular, when the demographics of the patients in this study are analysed, an MCL injury should be especially suspected in male patients and patients who might be considered older than the standard for patellar dislocation. In these patients, who may be considered outside the normal demographics for patellar dislocation, aggressive rehabilitation of the patellar dislocation injury should be treated with at least circumspection and should perhaps be avoided.

\section{Competing interests None.}

\section{REFERENCES}

1. Stefancin JJ, Parker RD. First-time traumatic patellar dislocation: a systematic review. Clin Orthop Relat Res 2007;455:93-101.

2. Harilainen A, Myllynen P, Antila $\mathrm{H}$, et al. The significance of arthroscopy and examination under anaesthesia in the diagnosis of fresh injury haemarthrosis of the knee joint. Injury 1988;19:21-4.

3. Hawkins RJ, Bell RH, Anisette G. Acute patellar dislocations. The natural history. Am J Sports Med 1986;14:117-20.

4. Cash JD, Hughston JC. Treatment of acute patellar dislocation. Am J Sports Med 1988:16:244-9.

5. Atkin DM, Fithian DC, Marangi KS, et al. Characteristics of patients with primary acute lateral patellar dislocation and their recovery within the first 6 months of injury. Am J Sports Med 2000;28:472-9.

6. King J. Patellar dislocation and lesions of the patella tendon. Br J Sports Med 2000:34:467-70.

7. Zeller BL, McCrory JL, Kibler WB, et al. Differences in kinematics and electromyographic activity between men and women during the single-legged squat. Am J Sports Med 2003;31:449-56.

8. Sallis RE, Jones K, Sunshine $\mathrm{S}$, et al. Comparing sports injuries in men and women. Int J Sports Med 2001;22:420-3.

9. Fithian DC, Paxton EW, Stone ML, et al. Epidemiology and natural history of acute patellar dislocation. Am J Sports Med 2004;32:1114-21.

10. Griffin LY, Agel J, Albohm MJ, et al. Noncontact anterior cruciate ligament injuries: risk factors and prevention strategies. J Am Acad Orthop Surg 2000;8:141-50.

11. Cowan SM, Bennell KL, Crossley KM, et al. Physical therapy alters recruitment of the vasti in patellofemoral pain syndrome. Med Sci Sports Exerc 2002:34:1879-85.

12. Elias DA, White LM. Imaging of patellofemoral disorders. Clin Radiol 2004;59:543-57.

13. Von Knoch F, Böhm T, Bürgi ML, et al. Trochleaplasty for recurrent patellar dislocation in association with trochlear dysplasia. A 4- to 14-year follow-up study. $J$ Bone Joint Surg Br 2006;88:1331-5.

14. Steiner TM, Torga-Spak R, Teitge RA. Medial patellofemoral ligament reconstruction in patients with lateral patellar instability and trochlear dysplasia. Am J Sports Med 2006;34:1254-61.
15. Simmons E Jr, Cameron JC. Patella alta and recurrent dislocation of the patella. Clin Orthop Relat Res 1992;274:265-9.

16. Huberti HH, Hayes WC. Patellofemoral contact pressures. The influence of q-angle and tendofemoral contact. J Bone Joint Surg Am 1984:66:715-24.

17. Healy WL, Anglen JO, Wasilewski SA, et al. Distal femoral varus osteotomy. J Bone Joint Surg Am 1988;70:102-9.

18. Rünow A. The dislocating patella. Etiology and prognosis in relation to generalized joint laxity and anatomy of the patellar articulation. Acta Orthop Scand Suppl 1983;201:1-53.

19. Feller JA, Amis AA, Andrish JT, et al. Surgical biomechanics of the patellofemoral joint. Arthroscopy 2007;23:542-53.

20. Karst GM, Willett GM. Onset timing of electromyographic activity in the vastus medialis oblique and vastus lateralis muscles in subjects with and without patellofemoral pain syndrome. Phys Ther 1995;75:813-23.

21. Cowan SM, Bennell KL, Hodges PW, et al. Delayed onset of electromyographic activity of vastus medialis obliquus relative to vastus lateralis in subjects with patellofemoral pain syndrome. Arch Phys Med Rehabil 2001;82:183-9.

22. Yu JS, Popp JE, Kaeding CC, et al. Correlation of MR imaging and pathologic findings in athletes undergoing surgery for chronic patellar tendinitis. AJR Am J Roentgenol 1995:165:115-18.

23. Nikku R, Nietosvaara Y, Kallio PE, et al. Operative versus closed treatment of primary dislocation of the patella. Similar 2-year results in 125 randomized patients. Acta Orthop Scand 1997:68:419-23

24. Hinton RY, Sharma KM. Acute and patellar instability in the young athlete. Orthop Clin North Am 2003;34:385-96.

25. Koëter S, Parkvis D, Van Loon CJ, et al. Trochlear osteotomy for patellar instability: satisfactory minimum 2-year results in patients with dysplasia of the trochlea. Knee Surg Sports Traumatol Arthrosc 2007;15:228-32.

26. Fukushima K, Horaguchi T, Okano T, et al. Patellar dislocation: arthroscopic patellar stabilization with anchor sutures. Arthroscopy 2004;20:761-4.

27. Hautamaa PV, Fithian DC, Kaufman KR, et al. Medial soft tissue restraints in lateral patellar instability and repair. Clin Orthop Relat Res 1998;349:174-82.

28. Ahmad CS, Stein BE, Matuz D, et al. Immediate surgical repair of the medial patellar stabilizers for acute patellar dislocation. A review of eight cases. Am J Sports Med 2000;28:804-10

29. Schweitzer ME, Tran D, Deely DM, et al. Medial collateral ligament injuries: evaluation of multiple signs, prevalence and location of associated bone bruises, and assessment with MR imaging. Radiology 1995;194:825-9.

30. Hunter SC, Marascalco R, Hughston JC. Disruption of the vastus medialis obliquus with medial knee ligament injuries. Am J Sports Med 1983;11:427-31.

31. Cromwell F, Walsh J, Gormley J. A pilot study examining injuries in elite Gaelic footballers. Br J Sports Med 2000;34:104-8.

32. Bathgate A, Best JP, Craig G, et al. A prospective study of injuries to elite Australian rugby union players. Br J Sports Med 2002;36:265-9.

33. Hawkins RD, Hulse MA, Wikinson $\mathrm{C}$, et al. The association football medical research programme: an audit of injuries in professional football. $\mathrm{Br} J$ Sports Med 2001;35:43-7.

34. Brinker MR, O'Connor DP. Basic sciences. In Miller MD, ed. Review of orthopaedics. Philadelphia, PA: Elsevier, 2008:111.

35. Verrall GM, Slavotinek JP, Fon GT. Incidence of pubic bone marrow oedema in Australian rules football players: relation to groin pain. Br J Sports Med 2001:35:28-33.

36. Orchard J, Wood T, Seward H, Broad A. Comparison of injuries in elite senior and junior Australian football. J Sci Med Sport 1998;1:83-8.

37. Garvin GJ, Munk PL, Vellet AD. Tears of the medial collateral ligament: magnetic resonance imaging findings and associated injuries. Can Assoc Radiol J 1993:44:199-204.

38. Yao L, Dungan D, Seeger LL. MR imaging of tibial collateral ligament injury comparison with clinical examination. Skeletal Radiol 1994;23:521-4.

39. Rasenberg El, Lemmens JA, van Kampen A, et al. Grading medial collateral ligament injury: comparison of MR imaging and instrumented valgus-varus laxity testdevice. A prospective double-blind study. Eur J Radiol 1995;21:18-24.

40. Blankenbaker DG, De Smet AA, Fine JP. Is intra-articular pathology associated with MCL edema on MR imaging of the non-traumatic knee? Skeletal Radiol 2005;34:462-7.

41. Mirowitz SA, Shu HH. MR imaging evaluation of knee collateral ligaments and related injuries: comparison of T1-weighted, T2-weighted, and fat-saturated T2weighted sequences-correlation with clinical findings. J Magn Reson Imaging 1994:4:725-32.

42. Fetto JF, Marshall JL. Medial collateral ligament injuries of the knee: a rationale for treatment. Clin Orthop Relat Res 1978;132:206-18.

43. Indelicato PA. Non-operative treatment of complete tears of the medial collateral ligament of the knee. J Bone Joint Surg Am 1983;65:323-9.

44. Yoshiya S, Kuroda R, Mizuno K, et al. Medial collateral ligament reconstruction using autogenous hamstring tendons: technique and results in initial cases. Am J Sports Med 2005;33:1380-5.

45. Grood ES, Noyes FR, Butler DL, et al. Ligamentous and capsular restraints preventing straight medial and lateral laxity in intact human cadaver knees. J Bone Joint Surg Am 1981;63:1257-69. 